\title{
Algebraic Modification of Trapezoidal Fuzzy Numbers to Complete Fully Fuzzy Linear Equations System Using Gauss-Jacobi Method
}

\author{
Ahmad Syaiful Abidin*, Mashadi Mashadi, Sri Gemawati \\ Department of Mathematics, University of Riau, Pekanbaru, Indonesia \\ Email address: \\ Ahmadsyaifulabidin2707@gmail.com(A. S. Abidin), mashadi.mat@gmail.com (M. Mashadi), gemawati.sri@gmail.com (S. Gemawati) \\ ${ }^{*}$ Corresponding author
}

To cite this article:

Ahmad Syaiful Abidin, Mashadi Mashadi, Sri Gemawati. Algebraic Modification of Trapezoidal Fuzzy Numbers to Complete Fully Fuzzy Linear Equations System Using Gauss-Jacobi Method. International Journal of Management and Fuzzy Systems.

Vol. 5, No. 2, 2019, pp. 40-46. doi: 10.11648/j.ijmfs.20190502.12

Received: July 20, 2019; Accepted: August 19, 2019; Published: September 2, 2019

\begin{abstract}
This paper will discuss algebraic modification of trapezoidal fuzzy numbers with a general form of fully fuzzy numbers is $\tilde{A} \tilde{x}=\tilde{b}$ with $\tilde{A}$ is $\mathrm{n} \times \mathrm{n}$ fuzzy matrix, $\tilde{x}$ fuzzy vector, and $\tilde{b}$ unknown fuzzy vector. The concept used in this paper is define positive or negative fuzzy numbers determined by the area on the left side of the $\mathrm{x}$-axis and the right side of the $\mathrm{x}$-axis. Furthermore, the concept will be applied to the multiplication of two fully fuzzy trapezoidal numbers to produce a new algebra that can be applied to a system of linear equations. At the end, an example of multiplication two fully fuzzy trapezoidal numbers using the Gauss-Jacobi method will be given. As a result compatible number will be obtained.
\end{abstract}

Keywords: Fully Fuzzy Number, Trapezoidal, New Algebra, Gauss-Jacobi Method

\section{Introduction}

Linear equation system has an important role in solving mathematic problems, one of them is used to solve problems in the operation of fuzzy numbers. The general form of a fully fuzzy linear equation system is $A \tilde{x}=\tilde{b}$. The method can be used in solving linear equation system in fuzzy numbers is divided into direct and indirect methods. Completion of the fully fuzzy linear equation system discussed by other researchers which is Sri [5] discusses fuzzy trapezoidal numbers using the $\mathrm{QR}$ decomposition method. Kumar et al [9] applies a new method to the fuzzy trapezoidal number named Mehar method and $\mathrm{J}$ Kaur et al [11] has comentary on "calculating fuzzy inverse matrix using fuzzy linear syatem". The other researcher is A. Jafarian [2] discuss about new decomposition method for solving dual fully fuzzy linear system. Most researchers only examined the fully fuzzy trapezoidal linear equation which has only positive values [1-2, 4, 6-9] with results that are not compatible yet. For this reason, the author offers an algebraic alternative for fuzzy trapezoidal numbers to obtain a competent solution.

\section{Preliminaries}

The concept of fuzzy sets was introduced by various authors [8, 10-13] and the gauss-jacobi method $[1,8]$.

Definition 2.1 Fuzzy number is a fuzzy set $\tilde{u}: R \rightarrow[0,1]$ which satisfies the following:

a) $\tilde{u}(x)$ upper semicontinous.

b) $\tilde{u}(x)=0$ outside the interval $[a-\alpha, b+\beta]$.

c) There exist real number $\mathrm{a}, \mathrm{b}$ in interval $[a-\alpha, b+\beta]$ such that,

i. $\tilde{u}(x)$ monotonic increasing in interval $[a-\alpha, a]$.

ii. $\tilde{u}(x)$ monotonic decreasing in interval $[b, b+\beta]$.

iii. $\tilde{u}(x)=1$ for $a \leq x \leq b$.

Definition 2.2 A Fuzzy number $\tilde{u}(x)$ in $\mathrm{R}$ are defined as function pair $(\underline{u}(r), \bar{u}(r))$ which statisfy the following:

a) $\underline{u}(r)$ is a bounded left continuous non decreasing function over $[0,1]$.

b) $\bar{u}(r)$ is a bounded left continuous non increasing function over $[0,1]$.

c) $\underline{u}(r) \leq \bar{u}(r), 0 \leq r \leq 1$.

Fuzzy trapezoidal number can be written in $\tilde{u}=$ 
$(a, b, \alpha, \beta)$ where $\mathrm{a}$ and $\mathrm{b}$ are the central points while $\alpha$ is distance from the point a left and $\beta$ is the distance from point $b$ to the right. These fuzzy numbers have membership functions as follows:

$$
\mu_{\widetilde{u}}(x)=\mu_{\widetilde{u}}(a, b, \alpha, \beta)=\left\{\begin{array}{c}
1-\frac{a-x}{\alpha}, a-\alpha \leq x \leq a \\
1, a \leq x \leq b \\
1-\frac{x-b}{\beta}, b \leq x \leq b+\beta \\
0, \text { otherwise }
\end{array}\right.
$$

Furthermore, in parametric form fuzzy number $\tilde{u}=$ $[\underline{u}(r), \bar{u}(r)]$ can be written with

$$
\begin{aligned}
& \underline{u}(r)=a-(1-r) \alpha \\
& \bar{u}(r)=b+(1-r) \beta
\end{aligned}
$$

Some authors have described arithmetic fuzzy trapezoidal numbers $[2-3,5,7,9,13-16]$. Two fuzzy number $\tilde{u}=$ $(a, b, \alpha, \beta)$ and $\tilde{v}=(c, d, \gamma, \delta)$ are equal if only if $a=c$, $b=d, \alpha=\gamma$, and $\beta=\delta$, or a fuzzy trapezoidal number is said to be zero fuzzy number if $a=c=0, b=d=0$, $\alpha=\gamma=0$, and $\beta=\delta=0$.

Operation of fuzzy numbers will be explained as follows:

Definition 2.3 arithmetic two fuzzy numbers $\tilde{u}=$ $(a, b, \alpha, \beta), \tilde{v}=(c, d, \gamma, \delta)$ and k scalar:

a) Addition

$$
\tilde{u} \oplus \tilde{v}=(a+c, b+d, \alpha+\gamma, \beta+\delta)
$$

b) Subtraction

$$
\tilde{u} \ominus \tilde{v}=(a-d, b-c, \alpha+\delta, \beta+\gamma)
$$

c) Scalar multiplication

$$
k \otimes \tilde{u}=k \otimes(a, b, \alpha, \beta)=\left\{\begin{array}{c}
(k a, k b, k \alpha, k \beta), k \geq 0 \\
(k b, k a,-k \beta,-k \alpha) k \leq 0
\end{array}\right.
$$

See that:

$$
-\tilde{u}=(-b,-a, \beta, \alpha)
$$

Before discussing arithmatic fuzzy numbers on the trapezoid need to be defined as positive fuzzy numbers and negative fuzzy numbers explained in the subsection below.

\section{Positive Fuzzy and Negative Fuzzy Numbers}

In this section a new definition will be given to determine a fuzzy number which is said to be positive fuzzy numbers or negative fuzzy numbers which will be use to modify algebra in multiplying two fuzzy numbers.

Fuzzy numbers are said to be positive (negative) if area $\mu_{\widetilde{u}}(x) \geq 0\left(\mu_{\widetilde{u}}(x)<0\right)$ or $L \geq 0(L<0)$ seen from the positive $\mathrm{x}$ and $\mathrm{x}$ negatives as follows:

a) If fuzzy numbers are only in the $x$-positive line area then it will be positive fuzzy number or $a-\alpha \geq 0$ and otherwise negative fuzzy number if $b+\beta<0$.

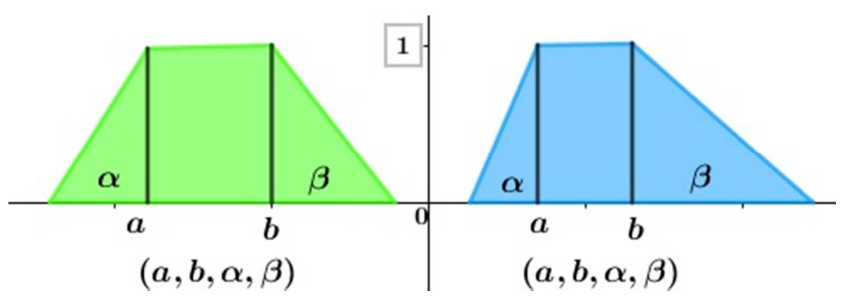

Figure 1. Illustration of negative and positive trapezoidal fuzzy numbers.

From the Figure 1 clearly visible area which is positife or negative.

b) If fuzzy is in both x-positive and X-negative areas, it is divided into 3 cases, as follows:

Case 1 Consider the following figure bellow

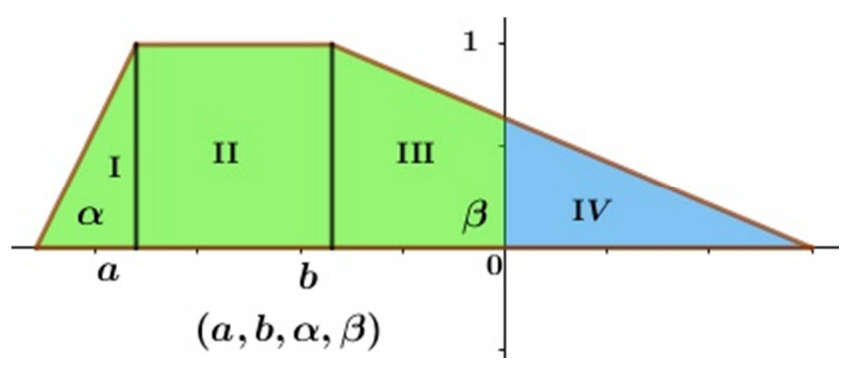

Figure 2. Illustration of case 1 for trapezoidal fuzzy numbers.

From the figure above, it can be seen that some areas are at $\mathrm{x}$-positive and others are at x-negative, so they can be divided into 4 (four) area sections, hence:

$$
\begin{gathered}
L_{I}=\frac{1}{2}(\alpha)(1)=\frac{\alpha}{2} \\
L_{I I}=(a-b)(1)=a-b \\
L_{I I I, I V}=\frac{1}{2}(\beta)(1)=\frac{\beta}{2} \\
L_{I V}=\frac{1}{2}(\beta+b)\left(\frac{\beta+b}{\beta}\right)=\frac{(\beta+b)^{2}}{2 \beta} \\
L_{I I I}=L_{I I I, I V}-L_{I V}=\frac{\beta}{2}-\frac{(\beta+b)^{2}}{2 \beta}=\frac{-2 \beta b-b^{2}}{2 \beta}
\end{gathered}
$$

Then overall area will be obtained, as follows:

$$
\begin{gathered}
L=L_{I V}-\left(L_{I}+L_{I I}+L_{I I I}\right) \\
L=\frac{(\beta+b)^{2}}{2 \beta}-\left(\frac{\alpha}{2}+a-b+\frac{\left(-2 \beta b-b^{2}\right)}{2 \beta}\right) \\
L=-a+b+\frac{\beta}{2}-\frac{\alpha}{2}+\frac{b^{2}}{\beta}+2 b
\end{gathered}
$$

If $a \leq 0, b \leq 0$ and $b+\beta \geq 0$, then $\tilde{u}$ positive fuzzy number if $-a-b+\frac{\beta}{2}-\frac{\alpha}{2}+\frac{b^{2}}{\beta} \geq 0$, otherwise $\tilde{u}$ negative fuzzy number if $-a+b+\frac{\beta}{2}-\frac{\alpha}{2}+\frac{b^{2}}{\beta}+2 b<0$.

Case 2 Consider the following figure bellow 


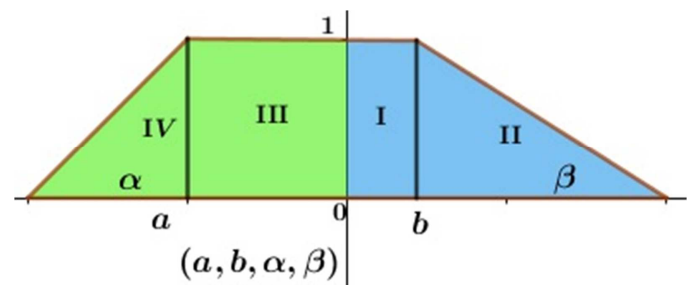

Figure 3. Illustration of case 2 for trapezoidal fuzzy numbers.

From the figure, similar way from case 1 it can be divided into 4 parts to find the area, hence

$$
\begin{gathered}
L_{I}=(b)(1)=b \\
L_{I I}=\frac{1}{2}(\beta)(1)=\frac{\beta}{2} \\
L_{I I I}=(a)(1)=a \\
L_{I V}=\frac{1}{2}(\alpha)(1)=\frac{\alpha}{2}
\end{gathered}
$$

Then overall area will be obtained, as follows:

$$
\begin{gathered}
L=\left(L_{I}+L_{I I}\right)-\left(L_{I I I}+L_{I V}\right) \\
L=\left(b+\frac{\beta}{2}\right)-\left(a+\frac{\alpha}{2}\right) \\
L=b-a+\frac{\beta}{2}-\frac{\alpha}{2}
\end{gathered}
$$

Therefore, conclusion is if $a \leq 0$ and $b \geq 0$, then $\tilde{u}$ positive fuzzy number if $b-a-\frac{\alpha}{2}+\frac{\beta}{2} \geq 0$, otherwise negative fuzzy number if $b-a-\frac{\alpha}{2}+\frac{\beta}{2}<0$.

Case 3 Consider the following figure bellow

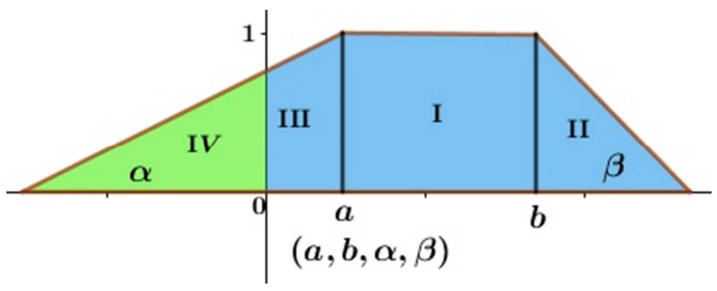

Figure 4. Illustration of case 3 for trapezoidal fuzzy numbers.

From the figure, similar way from case 1 and 2 it can be divided into 4 parts to find the area, hence

$$
\begin{gathered}
L_{I}=(b-a)(1)=b-a \\
L_{I I}=\frac{1}{2}(\beta)(1)=\frac{\beta}{2} \\
L_{I I I, I V}=\frac{1}{2}(\alpha)(1)=\frac{\alpha}{2} \\
L_{I V}=\frac{1}{2}(\alpha-a)\left(\frac{\alpha-a}{\alpha}\right)=\frac{(\alpha-a)^{2}}{2 \alpha} \\
L_{I I I}=L_{I I I, I V}-L_{I V}=\frac{\alpha}{2}-\frac{(\alpha-a)^{2}}{2 \alpha}=a-\frac{a^{2}}{2 \alpha}
\end{gathered}
$$

Then overall area will be obtained, as follows:

$$
\begin{gathered}
L=\left(L_{I}+L_{I I}+L_{I I I}\right)-\left(L_{I V}\right) \\
L=\left(b-a+\frac{\beta}{2}+a-\frac{a^{2}}{2 \alpha}\right)-\left(\frac{(\alpha-a)^{2}}{2 \alpha}\right) \\
L=a+b-\frac{\alpha}{2}+\frac{\beta}{2}-\frac{a^{2}}{\alpha}
\end{gathered}
$$

Futhermore, the conclusions is if $a \geq 0$ and $b \geq 0$, then $\tilde{u}$ positive fuzzy number if $a+b-\frac{\alpha}{2}+\frac{\beta}{2}-\frac{a^{2}}{\alpha} \geq 0$, otherwise negative fuzzy number if $a+b-\frac{\alpha}{2}+\frac{\beta}{2}-\frac{a^{2}}{\alpha}<0$.

After defining positive fuzzy numbers and negative fuzzy numbers and operation of fuzzy numbers then it will be applied to the multiplication of two fuzzy numbers will be explained in the following below:

Theorem 3.1 if $\tilde{u}=(\underline{u}(r), \bar{u}(r))$ and $\tilde{v}=(\underline{v}(r), \bar{v}(r))$ are two positiv fuzzy numbers, then $\widetilde{w}=\tilde{u} \otimes \tilde{v}=$ $(\underline{w}(r), \bar{w}(r))$, where

$$
\underline{w}(r)=\underline{u}(r) \underline{v}(1)+\underline{u}(1) \underline{v}(r)-\underline{u}(1) \underline{v}(1)
$$

For every $r \in[0,1]$ is a positive fuzzy numbers.

Based on the theorem 3.1 so we have definition below.

Definition 2.4 Let $\tilde{u}=(a, b, \alpha, \beta)=(\underline{u}(r), \bar{u}(r))=$ $(a-(1-r) \alpha, b+(1-r) \beta) \quad$ and $\quad \tilde{v}=(c, d, \gamma, \delta)=$ $(\underline{v}(r), \bar{v}(r))=(c-(1-r) \gamma, b+(1-r) \delta)$ be two fuzzy numbers, then:

a) If $\tilde{u}>0$ and $\tilde{v}>0$ then: $\tilde{w}=(a c, b d,(a \gamma+$ $c \alpha),(b \delta+d \beta))$

b) If $\tilde{u}>0$ and $\tilde{v}<0$ then: $\widetilde{w}=(b c, a d,(b \gamma-$ $c \beta),(a \delta-d \alpha))$

c) If $\tilde{u}<0$ and $\tilde{v}>0$ then: $\tilde{w}=(a d, b c,(d \alpha-$ $a \delta),(c \beta-b \gamma))$

d) If $\tilde{u}<0$ and $\tilde{v}<0$ then: $\widetilde{w}=(b d, a c,-(d \beta+$ $b \delta),-(a \gamma+c \alpha))$

Then, we will discusss of positive (negative) fuzzy numbers in the above definition using the Gauss-Jacobi method.

\section{Completion of Fully Fuzzy Linear Equation System}

In this section algebraic modifications will be given to multiply fuzzy numbers in a fully fuzzy linear equation system $\tilde{A} \tilde{x}=\tilde{b}$ by using definition 2.4. It begins by partitioning the fully fuzzy linear equation system which contains real matrices and real vector. Then it will be solved using the Gauss-Jacobi method. In this linear equation system we will find the value of $\tilde{x}$ from the positive (negative) definition of the matrix $\tilde{A}$ and vector $\tilde{b}$ then the formula used in definition 2.4.

Let $\tilde{A} \tilde{x}=\tilde{b}$ a fully fuzzy linear equation system with 


$$
\begin{gathered}
\tilde{A}=\left[\begin{array}{cccc}
\left(a_{11}, b_{11}, \alpha_{11}, \beta_{11}\right) & \left(a_{12}, b_{12}, \alpha_{12}, \beta_{12}\right) & \cdots & \left(a_{1 n}, b_{1 n}, \alpha_{1 n}, \beta_{1 n}\right) \\
\left(a_{21}, b_{21}, \alpha_{21}, \beta_{21}\right) & \left(a_{22}, b_{22}, \alpha_{22}, \beta_{22}\right) & \cdots & \left(a_{2 n}, b_{2 n}, \alpha_{2 n}, \beta_{2 n}\right) \\
\vdots & \vdots & & \ddots \\
\left(a_{m 1}, b_{m 1}, \alpha_{m 1}, \beta_{m 1}\right) & \left(a_{m 2}, b_{m 2}, \alpha_{12}, \beta_{12}\right) & \cdots & \left(a_{m n}, b_{m n}, \alpha_{m n}, \beta_{m n}\right)
\end{array}\right] \\
\tilde{x}=\left[\begin{array}{c}
\left(x_{1}, y_{1}, \gamma_{1}, \delta_{1}\right) \\
\left(x_{2}, y_{2}, \gamma_{2}, \delta_{2}\right) \\
\vdots \\
\left(x_{m}, y_{m}, \gamma_{m}, \delta_{m}\right)
\end{array}\right], \tilde{b}=\left[\begin{array}{c}
\left(b_{1}, g_{1}, \pi_{1}, \varepsilon_{1}\right) \\
\left(b_{1}, g_{1}, \pi, \varepsilon_{1}\right) \\
\vdots \\
\left(b_{m}, g_{m}, \pi_{m}, \varepsilon_{m}\right)
\end{array}\right]
\end{gathered}
$$

which can be formed into

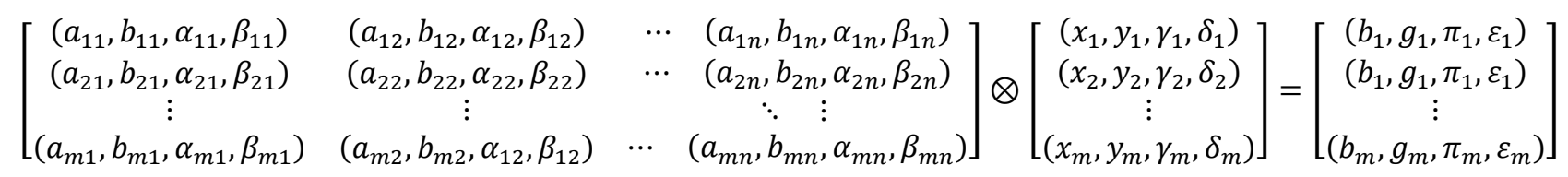

Fuzzy matrix $\tilde{A}$ is partitioned into

$A=\left[\begin{array}{cccc}a_{11} & a_{12} & \cdots & a_{1 n} \\ a_{21} & a_{22} & \cdots & a_{2 n} \\ \vdots & \vdots & \ddots & \vdots \\ a_{m 1} & a_{m 2} & \cdots & a_{m n}\end{array}\right], M=\left[\begin{array}{cccc}b_{11} & b_{12} & \cdots & b_{1 n} \\ b_{21} & b_{22} & \cdots & b_{2 n} \\ \vdots & \vdots & \ddots & \vdots \\ b_{m 1} & b_{m 2} & \cdots & b_{m n}\end{array}\right]$
$N=\left[\begin{array}{ccccc}\alpha_{11} & \alpha_{12} & \cdots & \alpha_{1 n} \\ \alpha_{21} & \alpha_{22} & \cdots & \alpha_{2 n} \\ \vdots & \vdots & \ddots & \vdots \\ \alpha_{m 1} & \alpha_{m 2} & \cdots & \alpha_{m n}\end{array}\right], K=\left[\begin{array}{cccc}\beta_{11} & \beta_{12} & \cdots & \beta_{1 n} \\ \beta_{21} & \beta_{22} & \cdots & \beta_{2 n} \\ \vdots & \vdots & \ddots & \vdots \\ \beta_{m 1} & \beta_{m 2} & \cdots & \beta_{m n}\end{array}\right]$

Fuzzy matrix $\tilde{A}$ can be written $\tilde{A}=(A, M, N, K)$.

Then fuzzy vector $\tilde{x}$ is partitioned into

$$
x=\left[\begin{array}{c}
x_{1} \\
x_{2} \\
\vdots \\
x_{m}
\end{array}\right] y=\left[\begin{array}{c}
y_{1} \\
y_{2} \\
\vdots \\
y_{m}
\end{array}\right] \gamma=\left[\begin{array}{c}
\gamma_{1} \\
\gamma_{2} \\
\vdots \\
\gamma_{m}
\end{array}\right] \delta=\left[\begin{array}{c}
\delta_{1} \\
\delta_{2} \\
\vdots \\
\delta_{m}
\end{array}\right]
$$

Hence, fuzzy vector $\tilde{x}$ can be written as $\tilde{x}=(x, y, \gamma, \delta)$.

Futhermore, fuzzy vector $\tilde{b}$ is partitioned into

$$
\left\{\begin{array}{c}
a_{i 1} x_{1}+a_{i 2} x_{2}+\cdots+a_{i n} x_{n}=b_{i} \\
m_{i 1} y_{1}+m_{i 2} y_{2}+\cdots+m_{i n} y_{n}=g_{i} \\
\left(a_{i 1} \gamma_{1}+a_{i 2} \gamma_{2}+\cdots+a_{i n} \gamma_{n}\right)+\left(n_{i 1} x_{1}+n_{i 2} x_{2}+\cdots+n_{i n} x_{n}\right)=\pi_{i} \\
\left(m_{i 1} \delta_{1}+m_{i 2} \delta_{2}+\cdots+m_{i n} \delta_{n}\right)+\left(k_{i 1} y_{1}+k_{i 2} y_{2}+\cdots+k_{i n} y_{n}\right)=\varepsilon_{i}
\end{array}\right.
$$

Hence,

$$
\left\{\begin{array}{c}
x_{i}=\frac{1}{a_{i i}}\left(b_{i}-\sum_{j=1, j \neq i}^{n} a_{i j} x_{j}\right) \\
y_{i}=\frac{1}{m_{i i}}\left(g_{i}-\sum_{j=1, j \neq i}^{n} m_{i j} y_{j}\right) \\
\gamma_{i}=\frac{1}{a_{i i}}\left(\pi_{i}-\left(\sum_{j=1, j \neq i}^{n} a_{i j} \gamma_{j}-\sum_{j=1}^{n} n_{i j} x_{j}\right)\right), 1 \leq i \leq n \\
\delta_{i}=\frac{1}{m_{i i}}\left(\varepsilon_{i}-\left(\sum_{j=1, j \neq i}^{n} m_{i j} \delta_{j}-\sum_{j=1}^{n} k_{i j} y_{j}\right)\right)
\end{array}\right.
$$

Then fuzzy vector $\tilde{b}$ can be written $\tilde{b}=(b, g, \pi, \varepsilon)$.

Moreover the linear equations system can be formed into

$$
\tilde{A} \tilde{x}=\tilde{b}
$$

$$
(A, M, N, K) \otimes(x, y, \gamma, \delta)=(b, g, \pi, \varepsilon) .
$$

The algebra is shown below

For multiplication $\tilde{A}>0, \tilde{b}>0$ and $\tilde{x}>0$, then

$$
\left\{\begin{array}{c}
A x=b \\
M y=g \\
A \gamma+N x=\pi \\
M \delta+K y=\varepsilon
\end{array}\right.
$$

Next, for $1 \leq i \leq n$ we have 
When written in matrix it becomes

$$
\left[\begin{array}{l}
x \\
y \\
\gamma \\
\delta
\end{array}\right]=J\left[\begin{array}{l}
x \\
y \\
\gamma \\
\delta
\end{array}\right]+\lambda
$$

To complete the above equation, the initial iteration value is determined $x^{(0)}, y^{(0)}, \gamma^{(0)}, \delta^{(0)}$ which will give more results $x^{(1)}, y^{(1)}, \gamma^{(1)}, \delta^{(1)}$. This process will continue until you get the results of a convergent statement. The

$$
\left\{\begin{array}{c}
x_{i}{ }^{(k+1)}=\frac{1}{a_{i i}}\left(b_{i}-\sum_{j=1, j \neq i}^{n} a_{i j} x_{j}^{k}\right) \\
y_{i}{ }^{(k+1)}=\frac{1}{m_{i i}}\left(g_{i}-\sum_{j=1, j \neq i}^{n} m_{i j} y_{j}^{k}\right) \\
\gamma_{i}{ }^{k+1)}=\frac{1}{a_{i i}}\left(\pi_{i}-\left(\sum_{j=1, j \neq i}^{n} a_{i j} \gamma_{j}^{k}-\sum_{j=1}^{n} n_{i j} x_{j}^{k}\right)\right), 1 \leq i \leq n \\
\delta_{i}{ }^{(k+1)}=\frac{1}{m_{i i}}\left(\varepsilon_{i}-\left(\sum_{j=1, j \neq i}^{n} m_{i j} \delta_{j}^{k}-\sum_{j=1}^{n} k_{i j} y_{j}^{k}\right)\right)
\end{array}\right.
$$

If the above equation is changed to a matrix then

$$
\left[\begin{array}{c}
x_{1}^{(k+1)} \\
x_{2}^{(k+1)} \\
\vdots \\
x_{n}^{(k+1)}
\end{array}\right]=\left[\begin{array}{cccc}
1 & \ldots & \ldots & \ldots \\
a_{11} & \ldots & & \\
& 1 & \ldots & \ldots \\
\cdots & \frac{a_{22}}{2} & \ddots & \\
\ldots & \ldots & \ddots & \vdots \\
\cdots & \ldots & \ldots & \frac{1}{a_{n n}}
\end{array}\right]\left(\left[\begin{array}{c}
b_{1} \\
b_{2} \\
\vdots \\
b_{n}
\end{array}\right]-\left[\begin{array}{cccc}
0 & a_{12} & \ldots & a_{1 n} \\
a_{21} & 0 & \ldots & a_{2 n} \\
\vdots & \vdots & \ddots & \vdots \\
a_{n 1} & a_{n 2} & \ldots & 0
\end{array}\right]\left[\begin{array}{c}
x_{1}{ }^{(k)} \\
x_{2}{ }^{(k)} \\
\vdots \\
x_{n}{ }^{(k)}
\end{array}\right]\right)
$$

Hence,

$$
\begin{gathered}
x^{(k+1)}=\left(D_{A}\right)^{-1} b-\left(D_{A}\right)^{-1}\left(L_{A}+U_{A}\right) x^{k} \\
y^{(k+1)}=\left(D_{M}\right)^{-1} g-\left(D_{M}\right)^{-1}\left(L_{M}+U_{M}\right) y^{k} \\
\gamma^{(k+1)}=\left(D_{A}\right)^{-1} \pi-\left(D_{A}\right)^{-1}\left(L_{A}+U_{A}\right) \gamma^{k}-\left(D_{A}\right)^{-1} N x^{k} \\
\delta^{(k+1)}=\left(D_{M}\right)^{-1} \varepsilon-\left(D_{M}\right)^{-1}\left(L_{M}+U_{M}\right) \delta^{k}-\left(D_{M}\right)^{-1} K y^{k}
\end{gathered}
$$

In similar way if $\tilde{A}>0$ and $\tilde{b}<0$ then $\tilde{x}<0$, hence

$$
\begin{gathered}
x^{(k+1)}=\left(D_{M}\right)^{-1} b-\left(D_{M}\right)^{-1}\left(L_{M}+U_{M}\right) x^{k} \\
y^{(k+1)}=\left(D_{A}\right)^{-1} g-\left(D_{A}\right)^{-1}\left(L_{A}+U_{A}\right) y^{k} \\
\gamma^{(k+1)}=\left(D_{M}\right)^{-1} \pi-\left(D_{M}\right)^{-1}\left(L_{M}+U_{M}\right) \gamma^{k}+\left(D_{M}\right)^{-1} K x^{k} \\
\delta^{(k+1)}=\left(D_{A}\right)^{-1} \varepsilon-\left(D_{A}\right)^{-1}\left(L_{A}+U_{A}\right) \delta^{k}+\left(D_{A}\right)^{-1} N y^{k}
\end{gathered}
$$

In similar way if $\tilde{A}<0$ and $\tilde{b}>0$ then $\tilde{x}<0$, hence

$$
\begin{gathered}
y^{(k+1)}=\left(D_{A}\right)^{-1} b-\left(D_{A}\right)^{-1}\left(L_{A}+U_{A}\right) y^{k} \\
x^{(k+1)}=\left(D_{M}\right)^{-1} g-\left(D_{M}\right)^{-1}\left(L_{M}+U_{M}\right) x^{k}
\end{gathered}
$$

Gauss-Jacobi method at the completion of a fully fuzzy linear equation system $\tilde{A} \tilde{x}=\tilde{b}$ convergent if and only if the Gauss-Jacobi method is converging to solve a linear equation system $A x=b$. If matrix $A$ on a linear equation system $A x=b$ has the dominant diagonal with $\left|a_{i i}\right|>$ $\sum_{j=1, j \neq i}^{n}\left|a_{i j}\right|, i=1,2,3, \ldots, n$ then iterations in the GaussJacobi convergent method at each $x^{(0)}, y^{(0)}, \gamma^{(0)}, \delta^{(0)}$.

Furthermore, when applied to the iteration method, thus$$
\delta^{(k+1)}=\left(D_{A}\right)^{-1} \pi+\left(D_{A}\right)^{-1}\left(L_{A}+U_{A}\right) \delta^{k}-\left(D_{A}\right)^{-1} N y^{k}
$$$$
\gamma^{(k+1)}=\left(D_{M}\right)^{-1} \varepsilon+\left(D_{M}\right)^{-1}\left(L_{M}+U_{M}\right) \gamma^{k}-\left(D_{M}\right)^{-1} K x^{k}
$$$$
\text { In similar way if } \tilde{A}<0 \text { and } \tilde{b}<0 \text { then } \tilde{x}>0 \text {, hence }
$$

$$
\begin{gathered}
y^{(k+1)}=\left(D_{M}\right)^{-1} b-\left(D_{M}\right)^{-1}\left(L_{M}+U_{M}\right) y^{k} \\
x^{(k+1)}=\left(D_{A}\right)^{-1} g-\left(D_{A}\right)^{-1}\left(L_{A}+U_{A}\right) x^{k} \\
\delta^{(k+1)}=\left(D_{M}\right)^{-1} \pi+\left(D_{M}\right)^{-1}\left(L_{M}+U_{M}\right) \delta^{k}+\left(D_{M}\right)^{-1} K y^{k} \\
\gamma^{(k+1)}=\left(D_{A}\right)^{-1} \varepsilon+\left(D_{A}\right)^{-1}\left(L_{A}+U_{A}\right) \gamma^{k}+\left(D_{A}\right)^{-1} N x^{k}
\end{gathered}
$$

On completion, a multiplication example will be given $\tilde{A}<0$ and $\tilde{b}<0$ with $\tilde{x}>0$ using Gauss-Jacobi method. The multiplication formula used if $\tilde{A}<0$ and $\tilde{b}>0$ as follow

$$
(A, M, N, K) \otimes(x, y, \gamma, \delta)=(b, g, \pi, \varepsilon)
$$

$$
A y, M x,(N y-A \delta),(K x-M \gamma)=(b, g, \pi, \varepsilon)
$$

Sample questions are given as follows: 
$(-8,-6,4,4) \tilde{x}_{1} \oplus(-2,-1,3,2) \tilde{x}_{2}=(-48,-18,78,36)$

$(-4,-3,1,2) \tilde{x}_{1} \oplus(-6,-5,5,4) \tilde{x}_{2}=(-64,-36,78,54)$

Then,

$$
\begin{gathered}
A y=b \\
M x=g \\
N y-A w=\pi \\
K x-M \gamma=\varepsilon
\end{gathered}
$$

It is known that the initial part of this iteration method is $x^{(0)}=(0,0), y^{(0)}=(0,0), \gamma^{(0)}=(0,0), \delta^{(0)}=(0,0)$.

Then we will find the value $(x, y, \gamma, \delta)$.

$$
\begin{gathered}
A y=b \\
{\left[\begin{array}{ll}
-8 & -2 \\
-4 & -6
\end{array}\right]\left[\begin{array}{l}
y_{1} \\
y_{2}
\end{array}\right]=\left[\begin{array}{l}
-48 \\
-64
\end{array}\right]}
\end{gathered}
$$

When applied to the iteration method, it is obtained

$$
\begin{aligned}
& y_{1}^{(k+1)}=\frac{1}{8}\left(48-2 y_{2}^{k}\right) \\
& y_{2}^{(k+1)}=\frac{1}{6}\left(64-4 y_{1}^{k}\right)
\end{aligned}
$$

Then

Table 1. Iteration for $y_{1}$ and $y_{2}$.

\begin{tabular}{lll}
\hline iteration & $\mathbf{y}_{\mathbf{1}}$ & $\mathbf{y}_{\mathbf{2}}$ \\
\hline 0 & 0 & 0 \\
1 & 6 & 10,66666667 \\
2 & 3,333333333 & 6,666666667 \\
3 & 4,333333333 & 8,4444444444 \\
4 & 3,888888889 & 7,777777778 \\
5 & 4,055555556 & 8,074074074 \\
$\ldots$ & $\ldots$ & $\ldots$ \\
16 & 3,999985711 & 7,999971422 \\
17 & 4,000007144 & 8,000009526 \\
18 & 3,999997619 & 7,999995237 \\
19 & 4,000001191 & 8,000001588 \\
20 & 3,999999603 & 7,999999206 \\
\hline
\end{tabular}

The results of the iteration obtained values $y=\left(y_{1}, y_{2}\right)=$ $(4,8)$.

Similar way for value $x$

$$
\begin{gathered}
M x=g \\
{\left[\begin{array}{ll}
-6 & -1 \\
-3 & -5
\end{array}\right]\left[\begin{array}{l}
x_{1} \\
x_{2}
\end{array}\right]=\left[\begin{array}{l}
-18 \\
-36
\end{array}\right]}
\end{gathered}
$$

When applied to the iteration method, thus

$$
\begin{aligned}
x_{1}^{(k+1)} & =\frac{1}{6}\left(18-x_{2}^{k}\right) \\
x_{2}^{(k+1)} & =\frac{1}{5}\left(36-3 x_{1}^{k}\right)
\end{aligned}
$$

Then,
Table 2. Iteration for $x_{1}$ and $x_{2}$.

\begin{tabular}{lll}
\hline Iteration & $\mathbf{x}_{\mathbf{1}}$ & $\mathbf{x}_{\mathbf{2}}$ \\
\hline 0 & 0 & 0 \\
1 & 3 & 7,2 \\
2 & 1,8 & 5,4 \\
3 & 2,1 & 6,12 \\
4 & 1,98 & 5,94 \\
5 & 2,01 & 6,012 \\
6 & 1,998 & 5,994 \\
7 & 2,001 & 6,0012 \\
8 & 1,9998 & 5,9994 \\
9 & 2,0001 & 6,00012 \\
$\ldots$ & $\ldots$ & $\ldots$ \\
16 & 1,9999999998 & 5,999999994 \\
17 & 2,00000001 & 6,000000012 \\
18 & 1,9999999998 & 5,999999994 \\
19 & 2,000000001 & 6,000000001 \\
20 & 2 & 5,999999999 \\
21 & 2 & 6 \\
\hline
\end{tabular}

The results of the iteration is values $x=\left(x_{1}, x_{2}\right)=(2,6)$.

Next, in similar way and substitute the values $x$ and yobtained values $\delta$ and $\gamma$.

$$
\begin{gathered}
N y-A \delta=\pi \\
{\left[\begin{array}{ll}
4 & 3 \\
1 & 5
\end{array}\right]\left[\begin{array}{l}
4 \\
8
\end{array}\right]-\left[\begin{array}{ll}
-8 & -2 \\
-4 & -6
\end{array}\right]\left[\begin{array}{l}
\delta_{1} \\
\delta_{2}
\end{array}\right]=\left[\begin{array}{l}
78 \\
78
\end{array}\right]}
\end{gathered}
$$

When applied to the iteration method, thus

$$
\begin{aligned}
& \delta_{1}^{(k+1)}=\frac{1}{8}\left(38-2 \delta_{2}^{k}\right) \\
& \delta_{2}^{(k+1)}=\frac{1}{6}\left(36-4 \delta_{1}^{k}\right)
\end{aligned}
$$

The results of the iteration obtained values $\delta=\left(\delta_{1}, \delta_{2}\right)=$ $(4,3)$.

And by substituting values $x$ then the value $\delta$ from the iteration $\gamma=\left(\gamma_{1}, \gamma_{2}\right)=(2,4)$.

\section{Conclusion}

In this paper the authors only discuss the definition already known using area of trapezoidal to get positive fuzzy and negative fuzzy numbers and then aplplied to Gauss-Jacobi method to produce a compatible solution with obtained trapezoidal fuzzy number by applying alternative fuzzy algebra on fully fuzzy equation system $\tilde{A} \tilde{x}=\tilde{b}$ with $\tilde{A}$ positive or negative $n \times n$ fuzzy matrix, $\tilde{x}$ and $\tilde{b}$ fuzzy vector. Therefore, further discussing could be focused on triangular or hexagonal to get a compatible solution for positive and negative fuzzy number.

\section{Acknowledgements}

I would like to express my gratitude to my advisor, Prof. Mashadi, Dr. M. D. H. Gamal and my mentors for their encouragement, constant support, helpful remarks and insightful comments on the paper. 


\section{References}

[1] T. Allahviranloo, S. Salahshour, M. Homayoun-Nejad dan D. Beleanu, General solution of fully fuzzy systems, Abstract and Applied Analysis, 2013, 2013, 1-9.

[2] A. jafarian, New decomposition method for solving dual fully fuzzy linear system, Imt. Journal Fuzzy Computation and Modelling, 2, 2016, 76-85.

[3] M. Deghan dan B. Hashemi, Iterative solution of fuzzy linear system, Applied Mathematics and Computation, 175, 2006, 645-674.

[4] S. Gemawati, Nasfianti, Mashadi dan A Hadi, A new for dual fully fuzzy linear system with trapezoidal fuzzy number by QR decomposition, Journal of Physics, 1116, 2018, 1-5.

[5] G. Malkawi, N. Ahmad dan H. Ibrahim, Solving fully fuzzy linear sys tem with the necessary an suficient to have a positive solution, Applied Mathematics and Information Sciences, 8, 2014, 1003-1019.

[6] A. Hadi, Mashadi dan S. Gemawati, On fuzzy n-inner product spaces, Journal of Physics, 020010, 2017, 1-6.

[7] S. I. Marni, Mashadi, S. Gemawati, Solving dual fully fuzzy linear system by use factorizations of the coeficient matrix for trapezoidal fuzzy number, Bulletin of Mathematics, 2, 2018, 145-156.

[8] N. J. Karthik dan E. Chandrasekaran, Solving fully fuzzy linear system with trapezoidal fuzzy number matrices by partitioning, International Jo- urnal of Computer Applications, 64, 2013, 35-38.
[9] A. Kumar, Neetu, dan A. Bansal, A new method to solve fully fuzzy linear system with trapezoidal fuzzy number, Canadian Journal on Science and Engineering Mathematics, 1, 2010, 45-56.

[10] A. Kumar, J. Kaur dan P singh, A new method for solving fuzzy linier programs with trapezoidal fuzzy numbers, Journal of Fuzzy, 2011, 2011, 1-12.

[11] A. Kumar and J. Kaur, Comentary on "Calculating fuzzy inverse matrix using fuzzy linear equation system", Applied Soft Computing, 58, 2017, 324-327.

[12] L. A. Zadeh, Fuzzy Sets, Information and Control, 8, 1965, 338-353.

[13] L. Abdullah dan N. A. Rahman, Jacobi-based methods in solving fuzzy linier system, International Journal of Mathematical and Computational Science, 7, 2013, 402-408.

[14] Mashadi, A new method for dual fully fuzzy linier system by use LU factorizations of the coeficient matrix, Jurnal Matematika dan Sains, 15, 2010, 101-106.

[15] S. Moloudzadeh, P. Darabi dan H. Khandani, The pseudo invers matrices to solve general fully fuzzy linear system, Journal of Soft Computing and Applications, 2013, 2013, 111 .

[16] S. H. Nasheri dan M. Gholami, Linear system of equation with trapezoidal fuzzy numbers, The Journal of Mathematics and Computer Science, 3, 2011, 71-79.

[17] S. Das dan S. Chakraverty, Numerical solution of fuzzy system of linear equation, Applications and Applied Mathematics, 7, 2012, 334-356. 